DFPD02/TH/14

EDO-EP-44

hep-th/0206104

June, 2002

\title{
The Superembedding Origin of the Berkovits Pure Spinor Covariant Quantization of Superstrings
}

\author{
Marco Matone $^{a, b}$, Luca Mazzucato $^{a}$, Ichiro Oda $^{c}$, Dmitri Sorokin $^{a, b, d}$ and Mario Tonin ${ }^{a, b}$ \\ ${ }^{a}$ Dipartimento di Fisica, Università degli Studi di Padova, \\ Via F. Marzolo 8, 35131 Padova, Italy \\ ${ }^{b}$ Istituto Nazionale di Fisica Nucleare, Sezione di Padova \\ ${ }^{c}$ Edogawa University, 474 Komaki, Nagareyama City, Chiba 270-0198, Japan \\ ${ }^{d}$ Institute for Theoretical Physics, NSC KIPT, 61108 Kharkov, Ukraine
}

\begin{abstract}
We show that the pure spinor formalism proposed by Berkovits to covariantly quantize superstrings is a gauge fixed, twisted version of the complexified $n=2$ superembedding formulation of the superstring. This provides the Berkovits approach with a geometrical superdiffeomorphism invariant ground. As a consequence, the absence of the worldsheet (super)diffeomorphism ghosts in the pure spinor quantization prescription and the nature of the Berkovits BRST charge and antighost are clarified. Since superembedding is classically equivalent to the Green-Schwarz formulation, we thus also relate the latter to the pure spinor construction.
\end{abstract}




\section{Introduction}

The superembedding is a geometrical description of the dynamics of superbranes by means of a specific embedding of worldvolume supersurfaces into target superspaces. It has proved to be a powerful method for studying various aspects of superbranes at the classical level (see [1] for a review). For instance, the superembedding description has explained the geometrical nature of the $\kappa$-symmetry of the Green-Schwarz formulation [2], which turns out to be a conventional extended local supersymmetry of the embedded superworldvolume (as was first proved for superparticles in [3, 4], for superstrings in [5]-[10] and then for all the other superbranes [11, 12, 13]). An original purpose of the superembedding approach has been to use the worldvolume supersymmetry nature of the $\kappa$-symmetry for making progress in solving a long-standing problem of the covariant quantization of the Green-Schwarz superstrings, the problem being just caused by the $\kappa$-symmetry of the latter. To this end doubly (worldsheet + target space) supersymmetric actions for superstrings were constructed in which the whole or part of the $\kappa$-symmetry was traded for a manifest irreducible worldsheet supersymmetry.円 In this way the superembedding encompasses properties of both the Green-Schwarz and the Neveu-Schwarz-Ramond description of the superstring. In the superembedding formulation the spinor coordinates $\theta^{\underline{\alpha}}(\xi)$ of superstrings (and in general of the superbranes) have worldvolume superpartners, auxiliary commuting spinor variables $\lambda \underline{\alpha}(\xi)$ which have the properties of twistors. This is why initially such formulations were called 'twistor-like'. In a formulation of the theory in terms of $n=2$ worldsheet superfields the conjugate spinors $\lambda \underline{\alpha}(\xi)$ and $\bar{\lambda} \underline{\alpha}(\xi)$ are complex and satisfy the pure spinor condition $\beth^{\natural} \lambda \underline{\underline{m}} \lambda=0=\bar{\lambda} \Gamma \underline{m} \bar{\lambda}$ (which is a part of the superembedding condition) [6, 8, 17], and $\theta \underline{\alpha}(\xi)$ also has a fermionic superpartner, an auxiliary field $\sigma^{\underline{\alpha}}$. Pure spinors have been considered as auxiliary fields in [18]. Later on, Howe derived superspace constraints in $D=10 \mathrm{SYM}$ and $D=10,11$ supergravity theories as integrability conditions along pure spinor lines [19].

The problem of the covariant quantization of the superstring using the 'twistor-like' formulation, and in particular most suitable $n=2$ supersymmetric pure spinor models, has been mainly assaulted by Berkovits [20]. p] Having started from superembedding techniques in [8], he has recently arrived at a pure spinor method of covariant quantization [24, 25, 26]. The pure spinor formalism has been also applied to superparticles, to a supermembrane [27] and to open superstrings [28]. In this long way a link with the original superembedding formulation has been lost somewhere.

In this paper we find and restore this link. Since the superembedding formulation is classically equivalent to the Green-Schwarz formulation we therefore also find a relation

\footnotetext{
${ }^{1}$ In earlier versions of doubly supersymmetric extended objects [14] the $\kappa$-symmetry remained an independent symmetry, and these models contained more degrees of freedom than conventional ones.

${ }^{2}$ Pure spinors were introduced by Cartan [15] and more recently have been considered also in [16].

${ }^{3}$ Related, though rather cumbersome, methods used Lorentz harmonics to insure covariance [21, 22, 23.
} 
between the reparametrization invariant and $\kappa$-symmetric Green-Schwarz superstring action and the action of Berkovits, thus completing results earlier obtained in [29]. We do this with the example of the heterotic string. Substantially the Berkovits action is a complexified superembedding formulation gauge-fixed in a conventional BRST manner.

A key point in establishing the relation with the Berkovits method is to complexify the model. Note that this step does not double the physical degrees of freedom since the complex conjugate fields never appear in the action of the model. The complexification is useful for two reasons, it allows one to treat $\lambda$ and $\bar{\lambda}$ as independent fields and to perform the twisting of an $n=(0,2)$ superconformal system [30] associated with the superstring model. Namely, upon complexification, solving a part of the superembedding condition and gauge fixing the $\kappa$-symmetries involving $\bar{\lambda}$ and $\sigma$, one can express them in terms of other fields of the model thus removing $\bar{\lambda}$ and $\sigma$ as independent fields from the action. In this stage the model is still invariant under $\kappa$-symmetries acting on $\theta$ and $\lambda$ as well as under the $n=(0,2)$ superdiffeomorphisms whose gauge fixing gives rise to an associated $n=(0,2)$ superconformal system.

Twisting the $n=(0,2)$ superconformal system has two important consequences. Firstly, it shifts the conformal weights of the fields with a non vanishing $U(1) \mathrm{R}$ - charge and makes the superconformal algebra of currents anomaly free (i.e. its central charge vanishes). In particular, the central charge of the system of superdiffeomorphism ghosts, which originally is -6 , vanishes after the twist. This shall allow us to drop from the action the superdiffeomorphism ghost system which eventually would arise upon gauge fixing the superdiffeomorphisms. Finally, the remaining $\kappa$-symmetries are gauge fixed by a standard BRST recipe, i.e. by adding to the action the BRST variation of a suitable 'gauge fermion' and using as a BRST charge the twisted superconformal charge with positive ghost number (positive $\mathrm{R}$ - charge before the twist), as the twisting procedure prescribes. In this way one recovers the Berkovits action with a simple BRST charge of the form $Q_{B}=\oint \lambda \underline{\alpha} d_{\underline{\alpha}}$, where $d_{\underline{\alpha}}$ is a fermionic constraint that acts as a covariant spinor derivative on target space superfields. The cohomology of $Q_{B}$ has been proven to give the correct superstring spectrum [26]. Furthermore, the twisted superconformal current with a negative ghost number reproduces a correct composite ' $b$ '-field of the Berkovits approach. We thus show that the Berkovits pure spinor formulation is a gauge fixed and twisted version of the (complexified) superstring in the superembedding approach. This provides the Berkovits method with a geometrical superdiffeomorphism invariant ground and explains both the absence of the (super)diffeomorphism ghosts in the pure spinor quantization prescription and the nature of its BRST charge and antighost.

The paper is organized as follows. In Section 2 and 3 we introduce the action for the $D=10$ heterotic string in the superembedding formulation in terms of $n=(0,2)$ worldsheet superfields [8, 17], analyze its local symmetries (which include the $n=(0,2)$ superdiffeomorphisms and six $\kappa$-symmetries) and demonstrate how it is related to the Green-Schwarz action. Section 4 is devoted to a detailed analysis of the $n=2$ superconformal structure 
of the action. In Section 5 we consider the procedure that leads from the superembedding model to the pure spinor effective action by means of the complexification, the gauge fixing of the local symmetries and the twisting of the $n=2$ superconformal theory. In Section 6 we generalize the construction to the case of a supergravity and super-Yang-Mills background. Finally, in Section 7 we present conclusions and suggestions for further study.

\section{The $n=(0,2)$ superembedding formulation of the het- erotic string}

Let us start by assuming that the worldsheet of the heterotic string be an $n=(0,2)$ supersurface parametrized by two bosonic (light-cone) coordinates $\xi^{++}, \xi^{--}$and two complex conjugate fermionic coordinates $\eta^{-}, \bar{\eta}^{-}$collectively defined as $z^{M}=\left(\xi^{++}, \xi^{--}, \eta^{-}, \bar{\eta}^{-}\right)$. Here

each + and - corresponds to the $d=2$ Lorentz group weight $-\frac{1}{2}$ and $+\frac{1}{2}$ respectively. These become right- and left-sector conformal weights upon gauge fixing the worldsheet superdiffeomorphisms (we shall call them 'conformal weights'). Note that ' -- ' and ' ++ ' are the $d=2$ light-cone vector indices and ',-+ ' are the Majorana spinor indices). The worldsheet supersurface is embedded into an $N=1, D=10$ target superspace parametrized by ten bosonic coordinates $x \underline{\underline{m}}$ and by sixteen Majorana-Weyl fermionic coordinates $\theta \underline{\underline{\mu}}$ collec-

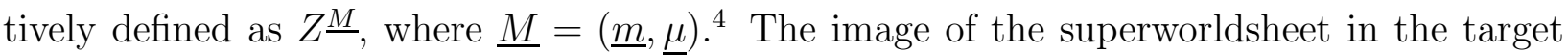
superspace is described by the $n=(0, \overline{2})$ superfields

$$
\begin{aligned}
& X^{\underline{m}}(z)=x^{\underline{m}}(\xi)+\eta^{-} \chi_{-}^{\underline{m}}(\xi)+\bar{\eta}^{-} \bar{\chi}_{-}^{\underline{m}}(\xi)+i \eta^{-} \bar{\eta}^{-} \nu_{--}^{\underline{m}}(\xi), \\
& \Theta^{\underline{\mu}}(z)=\theta \underline{\mu}(\xi)+\eta^{-} \lambda_{-}^{\underline{\mu}}(\xi)+\bar{\eta}^{-} \bar{\lambda}_{-}^{\underline{\mu}}(\xi)+i \eta^{-} \bar{\eta}^{-} \sigma_{-}^{\underline{\mu}}(\xi),
\end{aligned}
$$

whose leading components are the target superspace coordinates of the heterotic string. For the model to have the correct number of physical degrees of freedom, i.e. that of the heterotic string, the higher components of (2.1) and (2.2) should be auxiliary worldsheet fields. Therefore the superfields (2.1) and (2.2) should be constrained in a proper way. The constraint is the superembedding condition

$$
E_{-}^{\underline{a}}(Z \underline{\underline{M}}(z))=0=\bar{E}_{-}^{\underline{a}}(Z \underline{M}(z)),
$$

where $E_{-}^{\underline{a}}$ and $\bar{E}_{-}^{\underline{a}}$ are spinor components of the pullback onto the superworldsheet of the vector component of the target space supervielbein one form

$$
E^{\underline{A}}=e^{A}(z) E_{\bar{A}}^{\underline{A}}(Z(z))=e^{++} E_{++}^{\underline{A}}+e^{--} E_{--}^{\underline{A}}+e^{-} E_{-}^{\underline{A}}+\bar{e}^{-} \bar{E}_{-}^{\underline{A}},
$$

\footnotetext{
${ }^{4}$ Underlined and non underlined letters denote respectively target superspace and worldsheet superspace indices. Letters from the beginning and from the middle of the alphabet denote tangent superspace and supermanifold indices respectively. Of course, in flat superspace, curved and spinor indices may be identified. Also note that in our notation the complex conjugate of the product of two fermions is $\left(\psi_{1} \psi_{2}\right)^{*}=\bar{\psi}_{1} \bar{\psi}_{2}$.
} 
$e^{A}(z)$ being a worldsheet supervielbein one form and $\underline{A}=(\underline{a}, \underline{\alpha})$. For consistency the supervielbeins should satisfy worldsheet and target space supergravity constraints (see [1] and references therein for details).

In flat target superspace the superembedding condition on the pullback of the superinvariant form

$$
\Pi \underline{\underline{m}}=d X^{\underline{m}}-\frac{1}{2} d \Theta \Gamma \underline{\underline{m}} \Theta=e^{++} \Pi_{++}^{\underline{m}}+e^{--} \Pi_{\underline{-}}^{\underline{m}}+e^{-} \Pi \stackrel{\underline{m}}{-}+\bar{e}^{-} \bar{\Pi} \stackrel{\underline{m}}{-},
$$

where $d=e^{A} D_{A}$ is the worldsheet superspace differential, reduces to

$$
\Pi_{-}^{\underline{m}} \equiv D_{-} X^{\underline{m}}-\frac{1}{2} D_{-} \Theta \Gamma^{\underline{m}} \Theta=0, \quad \bar{\Pi}_{-}^{\underline{m}} \equiv \bar{D}_{-} X^{\underline{m}}-\frac{1}{2} \bar{D}_{-} \Theta \Gamma^{\underline{m}} \Theta=0,
$$

whose integrability requires the twistor-like constraint

$$
\Pi_{--}^{\underline{m}}=\frac{1}{2} D_{-} \Theta \Gamma^{\underline{m}} \bar{D}_{-} \Theta,
$$

and the pure spinor condition for the superfields $D_{-} \Theta$ and $\bar{D}_{-} \Theta$, that is

$$
D_{-} \Theta \Gamma^{\underline{m}} D_{-} \Theta=0, \quad \bar{D}_{-} \Theta \Gamma^{\underline{m}} \bar{D}_{-} \Theta=0 .
$$

The worldsheet supercovariant derivatives act as follows on a superfield $\Phi^{(+p,-q)}(z)$ of conformal weights $(+p,-q)$ (being, by definition, the weights of its leading component)

$$
\begin{gathered}
D_{-} \Phi^{(+p,-q)}=\left(\frac{\partial}{\partial \eta^{-}}+\bar{\eta}^{-} D_{--}\right) \Phi^{(+p,-q)}, \bar{D}_{-} \Phi^{(+p,-q)}=\left(\frac{\partial}{\partial \bar{\eta}^{-}}+\eta^{-} D_{--}\right) \Phi^{(+p,-q)}, \\
D_{--} \Phi^{(+p,-q)}=\left(\partial_{--}+e_{--}^{++} \partial_{++}+p \partial_{++} e_{--}^{++}\right) \Phi^{(+p,-q)}, \quad D_{++} \Phi^{(+p,-q)}=\partial_{++} \Phi^{(+p,-q)},
\end{gathered}
$$

$e_{--}^{++}(\xi)$ being a Beltrami parameter (i.e. the only nontrivial component of the worldsheet supervielbein (2.4)).

The superembedding condition (2.6) leads to the following relations between the components of the superfields (2.1) and (2.2)

$$
\begin{gathered}
\chi_{-}^{\underline{m}}-\frac{1}{2} \theta \Gamma^{\underline{m}} \lambda_{-}=0, \quad \bar{\chi}_{-}^{\underline{m}}-\frac{1}{2} \theta \Gamma^{\underline{m}} \bar{\lambda}_{-}=0, \quad \nu_{--}^{\underline{m}}-\frac{1}{2} \sigma_{--} \Gamma^{\underline{m}} \theta=0, \\
\lambda_{-} \Gamma^{\underline{m}} \lambda_{-}=0, \quad \bar{\lambda}_{-} \Gamma^{\underline{m}} \bar{\lambda}_{-}=0, \\
\left.\Pi_{--}^{\underline{m}}\right|_{\eta, \bar{\eta}=0}-\frac{1}{2} \lambda_{-} \Gamma^{\underline{m}} \bar{\lambda}_{-}=\partial_{--} x^{\underline{\underline{m}}}-\frac{1}{2} \partial_{--} \theta \Gamma^{\underline{m}} \theta-\frac{1}{2} \lambda_{-} \Gamma^{\underline{m}} \bar{\lambda}_{-}=0,
\end{gathered}
$$

${ }^{5}$ Analogous relations also hold in the curved supergravity background for the components of the target space supervielbein pullbacks. 


$$
\left(\partial_{--} \theta+i \sigma_{--}\right) \Gamma^{\underline{m}} \lambda_{-}=0, \quad\left(\partial_{--} \theta-i \sigma_{--}\right) \Gamma^{\underline{m}} \bar{\lambda}_{-}=0 .
$$

Eqs. (2.10)-(2.13), together with the local symmetries of the model, will allow us to express the higher components of the superfields (2.1) and (2.2) in terms of $x^{\underline{m}}$ and $\theta^{\underline{\alpha}}$ and their derivatives. Note that eq. 2.11) is the pure spinor condition for $\lambda_{-}^{\underline{\alpha}}$ and $\bar{\lambda} \underline{\alpha}$, while the twistor-like condition (2.12) implies one of the Virasoro constraints, namely

$$
\Pi_{--}^{\underline{m}} \Pi_{--\underline{m}}=0 \text {. }
$$

The fact that the supermebedding condition does not produce dynamical equations of motion for $x^{\underline{m}}$ and $\theta^{\underline{\alpha}}$ allows one to construct the $n=(0,2)$ worldsheet superfield action for the heterotic string [8] in an $N=1, D=10$ supergravity background

$$
I_{S E}=\int d^{2} \xi d \eta_{-} d \bar{\eta}_{-}\left[P_{++\underline{a}}^{-} E_{-}^{\underline{a}}+\bar{P}_{++\underline{a}}^{-} \bar{E}_{-}^{\underline{a}}+\frac{1}{2} \bar{\Psi}_{+}^{I} \Psi_{+}^{I}\right]+\frac{1}{2} \int d^{2} \xi\left[d \eta_{-} B_{++,-}+d \bar{\eta}_{-} \bar{B}_{++,-}\right] .
$$

In this action the superfields

$$
\begin{aligned}
& P_{++\underline{a}}^{-}(z)=\rho_{++\underline{a}}^{-}+\eta^{-} a_{++\underline{a}}+\bar{\eta}^{-} r_{++\underline{a}}+i \eta^{-} \bar{\eta}^{-} \rho_{-++\underline{a}}, \\
& \bar{P}_{++\underline{a}}^{-}(z)=\bar{\rho}_{++\underline{a}}^{-}+\bar{\eta}^{-} \bar{a}_{++\underline{a}}+\eta^{-} \bar{r}_{++\underline{a}}+i \eta^{-} \bar{\eta}^{-} \bar{\rho}_{-++\underline{a}},
\end{aligned}
$$

are complex conjugate Lagrange multipliers whose variation produces the superembedding condition (2.3) and, as a consequence, the Virasoro constraint (2.14). The second Virasoro constraint is obtained by varying the action with respect to the Beltrami parameter $e_{--}^{++}(\xi)$ (2.9). $\Psi_{+}^{I}(z)$ and $\bar{\Psi}_{+}^{I}(z)(I=1, \cdots, 16)$ are heterotic fermion (anti)chiral superfields (i.e. $\left.\bar{D}_{-} \Psi_{+}^{I}=0=D_{-} \bar{\Psi}_{+}^{I}\right)$, and $B_{++,-}, \bar{B}_{++,-}$are complex conjugate spin-vector components of the pullback onto the superworldsheet of the NS-NS two-form potential $B^{(2)}$. It can be shown that modulo the superembedding condition and the $N=1, D=10$ supergravity constraints, the superfields $B_{++,-}$and $\bar{B}_{++,-}$are chiral and antichiral respectively. For instance, in flat target superspace

$$
\begin{gathered}
B^{(2)}=d X^{\underline{m}} d \Theta \Gamma_{\underline{m}} \Theta=\Pi \underline{\underline{m}} d \Theta \Gamma_{\underline{m}} \Theta, \\
B_{++,-}=\Pi \frac{\underline{m}}{++} D_{-} \Theta \Gamma_{\underline{m}} \Theta-\Pi \frac{\underline{m}}{-} D_{++} \Theta \Gamma_{\underline{m}} \Theta, \\
\bar{B}_{++,-}=\Pi_{++}^{\underline{m}} \bar{D}_{-} \Theta \Gamma_{\underline{m}} \Theta-\bar{\Pi}_{-}^{\underline{m}} D_{++} \Theta \Gamma_{\underline{m}} \Theta,
\end{gathered}
$$

and

$$
D_{-} B_{++,-}=D_{++}\left(\Pi_{-}^{\underline{m}} D_{-} \Theta \Gamma_{\underline{m}} \Theta\right)-2 D_{-} \Pi_{-}^{\underline{m}} D_{++} \Theta \Gamma_{\underline{m}} \Theta-2 \Pi_{-}^{\underline{m}} D_{++} \Theta \Gamma_{\underline{m}} D_{-} \Theta,
$$

which vanishes when (2.6) is satisfied. 
By construction the target superspace covariant action (2.15) is invariant under worldsheet superdiffeomorphisms, even if it does not contain the worldsheet supervielbeins (apart from the Beltrami parameter $\left.e_{--}^{++}(\xi)\right)$, provided that the superbackground satisfies the $N=1$, $D=10$ supergravity constraints. Furthermore, the Lagrange multipliers $P_{++\underline{a}}^{-}, \bar{P}_{++\underline{a}}^{-}$must vary in a proper way to cancel the terms, proportional to the superembedding condition, coming from the variation of $B_{++,-}$and $\bar{B}_{++,-}$.

The superdiffeomorphism variations of the target superspace coordinates $Z \underline{M}(z)$ are

$$
\delta Z^{\underline{M}}=D_{-} \mathcal{C}^{--} \bar{D}_{-} Z^{\underline{M}}+\bar{D}_{-} \mathcal{C}^{--} D_{-} Z^{\underline{M}}+2 \mathcal{C}^{--} D_{--} Z^{\underline{M}}+2 c^{++} D_{++} Z^{\underline{M}},
$$

where $c^{++}(\xi)$ is a parameter of the left-sector bosonic reparametrizations and

$$
\mathcal{C}^{--}(z)=c^{--}(\xi)+\eta^{-} \bar{\gamma}^{-}(\xi)+\bar{\eta}^{-} \gamma^{-}(\xi)+i \eta^{-} \bar{\eta}^{-} v(\xi)
$$

is an unconstrained superfield parameter of the right-sector superdiffeomorphisms. In what follows we shall denote the BRST ghosts associated with the superdiffeomorphisms (2.20) with the same letters as in (2.21), and denote the corresponding antighosts by

$$
\mathcal{B}_{--}(z)=u_{--}(\xi)+\eta^{-} \beta_{---}(\xi)+\bar{\eta}^{-} \bar{\beta}_{---}(\xi)+i \eta^{-} \bar{\eta}^{-} b_{----}(\xi) \text {. }
$$

The odd components of the superdiffeomorphisms (2.20) replace two independent $\kappa^{-}$ symmetry transformations of the Green-Schwarz formulation. The remaining six nonmanifest $\kappa$-symmetries of the $D=10$ heterotic string action (2.15) are realized as follows

$$
\delta Z^{\underline{M}} E_{\underline{\underline{M}}}^{\underline{\alpha}}=E_{-} \Gamma^{\underline{a}} \bar{E}_{-}\left(\Gamma_{\underline{a}} K^{--}\right) \underline{\alpha}-2 E_{-}^{\underline{\alpha}} \bar{E}_{-} K^{--}-2 \bar{E}_{-}^{\underline{\alpha}} E_{-} K^{--}, \quad \delta Z^{\underline{M}} E_{\underline{M}}^{\underline{a}}=0
$$

where, among the sixteen fermionic superfield parameters $K_{\underline{\alpha}}^{--}(z)$ only six are independent. The action (2.15) is also invariant under the following local transformations of the Lagrange multipliers 8

$$
\delta P_{++\underline{a}}^{-}=D_{-} \Xi_{++}^{---} \Gamma_{\underline{a}} E_{-}, \quad \delta \bar{P}_{++\underline{a}}^{-}=\bar{D}_{-} \bar{\Xi}_{++}^{---} \Gamma_{\underline{a}} \bar{E}_{-},
$$

where among the complex conjugate superfield parameters $\Xi_{++}^{--\underline{\alpha}}(z)$ and $\bar{\Xi}_{++}^{--\underline{\alpha}}(z)$ only ten real are independent. The spinor supervielbein pullbacks $E_{-}^{\underline{\alpha}}$ and $\bar{E}_{-}^{\underline{\alpha}}$ reduce to $D_{-} \Theta^{\underline{\alpha}}$ and $\bar{D}_{-} \Theta^{\underline{\alpha}}$ in flat target superspace.

We now proceed with analyzing the action (2.15) and the superembedding condition in the flat superbackground and will consider its coupling to $N=1, D=10$ supergravity and super-Yang-Mills in Section 6.

\section{The Green-Schwarz action from superembedding}

Let us first perform the integration over the fermionic coordinates in the action (2.15). Next we solve for the auxiliary fields $\chi_{-}^{\underline{m}}, \bar{\chi}_{-}^{\underline{m}}$ and $\nu_{-}^{\underline{\alpha}}$ by means of (2.10). Then, eliminating the 
corresponding components of the Lagrange multipliers (2.16), using the equations of motion of $\chi_{-}^{\underline{m}}$ and $\bar{\chi}_{-}^{\underline{m}}$ and $(2.24)$, we obtain

$$
\begin{aligned}
I_{S E} & =\frac{1}{2} \int d^{2} \xi\left[\Pi_{++}^{\underline{m}} \Pi_{--\underline{m}}+\frac{1}{2} \Pi_{++}^{\underline{m}} D_{--} \theta \Gamma_{\underline{m}} \theta-\frac{1}{2} \Pi_{--}^{\underline{m}} D_{++} \theta \Gamma_{\underline{m}} \theta\right. \\
& \left.+i \bar{\psi}_{+}^{I} D_{--} \psi_{+}^{I}+i \psi_{+}^{I} D_{--} \bar{\psi}_{+}^{I}\right] \\
& +\int d^{2} \xi\left[p_{++\underline{m}}\left(\Pi_{--}^{\underline{m}}-\frac{1}{2} \lambda_{-} \Gamma^{\underline{m}} \bar{\lambda}_{-}\right)+r_{++\underline{m}} \lambda_{-} \Gamma \frac{\underline{m}}{\lambda_{-}+\bar{r}_{++\underline{m}}} \bar{\lambda}_{-} \Gamma^{\underline{m}} \bar{\lambda}_{-}\right. \\
& \left.+\rho_{++\underline{m}}^{-}\left(\partial_{--} \theta+i \sigma_{--}\right) \Gamma \underline{\underline{m}} \lambda_{-}+\bar{\rho}_{++\underline{m}}^{-}\left(\partial_{--} \theta-i \sigma_{--}\right) \Gamma \underline{m} \bar{\lambda}_{-}\right],
\end{aligned}
$$

where

$$
\Pi_{ \pm \pm}^{\underline{m}} \equiv D_{ \pm \pm} x^{\underline{m}}-\frac{1}{2} D_{ \pm \pm} \theta \Gamma_{\underline{m}} \theta,
$$

now stands for the leading components of the superfields (2.5), $\psi^{I}=\left.\Psi^{I}\right|_{\eta=\bar{\eta}=0}, \bar{\psi}^{I}=\left.\bar{\Psi}^{I}\right|_{\eta=\bar{\eta}=0}$, while $p_{++\underline{m}}=2 a_{++\underline{m}}+2 \bar{a}_{++\underline{m}}-\Pi_{++\underline{m}}$. Furthermore $r_{++\underline{m}}, \bar{r}_{++\underline{m}}, \rho_{++\underline{m}}^{-}$and $\bar{\rho}_{++\underline{m}}^{-}$are the remaining components of the Lagrange multipliers (2.16).

If we now solve the equations of motion of $\lambda_{-}, \bar{\lambda}_{-}$and $\sigma_{--}$, and gauge fix the local symmetries remaining in (2.24), we can set $r_{++\underline{m}}=\bar{r}_{++\underline{m}}=\rho_{++\underline{m}}^{-}=\bar{\rho}_{++\underline{m}}^{-}=0$ and find that

$$
p_{++\underline{m}}=e_{++}^{--}(\xi) \lambda_{-} \Gamma_{\underline{m}} \bar{\lambda}_{-} .
$$

Here $e_{++}^{--}(\xi)$ is a worldsheet parameter which can be regarded as the second component of the worldsheet vielbein, in addition to the Beltrami parameter $e_{--}^{++}(\xi)$ in (2.9).

The superfield counterpart of eq. (3.3) is

$$
D_{-}\left(P_{++\underline{m}}^{-}+\frac{1}{2} \eta^{-} \Pi_{++\underline{m}}\right)-\Pi_{++\underline{m}}=\frac{1}{2} \mathcal{E}_{++}^{--}(z) D_{-} \Theta \Gamma_{\underline{m}} \bar{D}_{-} \Theta \text { and c.c. },
$$

where $\mathcal{E}_{++}^{--}(z)$ is a superfield, containing $e_{++}^{--}(\xi)$ as the leading component, which can be regarded as a super-Beltrami parameter associated with the $n=(0,2), d=2$ superdiffeomorphisms.

Substituting (3.3) into (3.1), taking into account that $\left(\lambda_{-} \Gamma_{\underline{m}} \bar{\lambda}_{-}\right)^{2}=0$ because of the pure spinor condition, and using once again the twistor-like condition $\Pi_{--}^{\underline{m}}=\frac{1}{2} \lambda_{-} \Gamma_{\underline{m}} \bar{\lambda}_{-}$to eliminate $\lambda$ and $\bar{\lambda}$ from the action, we get the Green-Schwarz action for the heterotic string in the following form

$$
I_{G S}=\frac{1}{2} \int d^{2} \xi\left[e_{++}^{m} e_{--}^{n} \Pi \frac{m}{m} \Pi_{n \underline{m}}+\epsilon^{m n} \Pi_{m}^{\underline{m}} \partial_{n} \theta \Gamma_{\underline{m}} \theta+e_{--}^{m}\left(\bar{\psi}_{+}^{I} \partial_{m} \psi_{+}^{I}+\psi_{+}^{I} \partial_{m} \bar{\psi}_{+}^{I}\right)\right],
$$

where

$$
\Pi \frac{m}{m}=\partial_{m} x^{\underline{m}}-\frac{1}{2} \partial_{m} \theta \Gamma \underline{m} \theta
$$

and $e_{++}^{m}=\left(1-e_{++}^{--} e_{--}^{++}, e_{++}^{--}\right), e_{--}^{m}=\left(e_{--}^{++}, 1\right)\left(\right.$ notice that $\left.\operatorname{det} e_{a}^{m}=1\right)$. 
Let us note that the first integral in the action (3.1) coincides with the Green-Schwarz action (3.5) when in the latter the right-sector diffeomorphisms $\delta \xi^{--}=c^{--}(\xi)$ are gauge fixed by imposing (locally) the conformal gauge $e_{++}^{--}(\xi)=0$. Therefore, the superembedding action is basically the Green-Schwarz action gauge fixed in the right-sector plus the terms which ensure the superembedding condition and the full left-right (super)diffeomorphism invariance of the construction. We have thus demonstrated the classical equivalence of the superembedding and the Green-Schwarz formulation of the heterotic superstring.

\section{The $n=(0,2)$ superconformal structure of the su- perembedding action}

Let us now analyze the form of the first-class constraints generating the $n=(0,2), d=$ 2 superdiffeomorphisms which comprise the $U(1)$ current $j(\xi)$ of R-symmetry, two local supersymmetry currents $G(\xi)$ and $\bar{G}(\xi)$, and the energy momentum tensor $T(\xi)$ associated with the right-sector bosonic diffeomorphisms. ${ }^{6}$ These are conserved currents of matter fields. Furthermore, they are first class constraints of the model which form an $n=(0,2)$, $d=2$ supermultiplet that can be cast into the superfield

$$
J_{--}=j+i \eta^{-} G-i \bar{\eta}^{-} \bar{G}-2 i \eta^{-} \bar{\eta}^{-} T,
$$

whose explicit form can be found by the standard Noether procedure. To this end one considers the variation of the action (2.15) under $U(1) \mathrm{R}$-symmetry transformations of the Grassmann-odd coordinates $\delta \eta=i \phi \eta, \delta \bar{\eta}=-i \phi \bar{\eta}$ and regards $D_{++}$as the time derivative in order to identify the corresponding conjugate momenta of $X^{\underline{m}}$ and $\Theta^{\underline{\alpha}}$. Note that only the 'Wess-Zumino' $B^{(2)}$-term (2.18) of the action contributes to the definition of the supercurrent which, up to a square of the superembedding condition, has the form

$$
\begin{aligned}
J_{--} & =i\left(D_{-} \Theta W_{-}-\bar{D}_{-} \Theta \bar{W}_{-}\right)+i D_{-} X^{\underline{m}} \bar{D}_{-} X_{\underline{m}}-\frac{i}{4} D_{-} \Theta \Gamma_{\underline{m}} \Theta \bar{D}_{-} \Theta \Gamma_{\underline{m}} \Theta \\
& =i\left(D_{-} \Theta W_{-}-\bar{D}_{-} \Theta \bar{W}_{-}\right)+\frac{i}{4} \Pi_{-}^{\underline{m}} \bar{D}_{-} \Theta \Gamma_{\underline{m}} \Theta-\frac{i}{4} \bar{\Pi}_{-}^{\underline{m}} D_{-} \Theta \Gamma_{\underline{m}} \Theta .
\end{aligned}
$$

Here $W_{-\underline{\alpha}} \approx 0$ and $\bar{W}_{-\underline{\alpha}} \approx 0$ are complex conjugate chiral superfield constraints] containing the canonical momenta of the components of $\Theta^{\underline{\alpha}}$ and $\bar{\Theta}^{\underline{\alpha}}$. Namely

$$
W_{-\underline{\alpha}}=\omega_{-\underline{\alpha}}+\eta^{-}\left[d_{--\underline{\alpha}}-i D_{--} \tau_{\underline{\alpha}}+\frac{1}{2}\left(\Pi_{--}^{\underline{m}}-\frac{1}{2} \lambda_{-} \Gamma_{\underline{m}} \bar{\lambda}_{-}\right)\left(\Gamma^{\underline{m}} \theta\right)_{\underline{\alpha}}\right]+\eta^{-} \bar{\eta}^{-} D_{--} \omega_{-\underline{\alpha}},
$$

${ }^{6}$ To simplify notation we have omitted to specify the conformal weights of the currents $j, G, \bar{G}$, $T$, which are $1,3 / 2,3 / 2$, and 2 , respectively.

${ }^{7}$ The symbol $\approx$ indicates that the Hamiltonian constraints are in general satisfied in a weak sense. 


$$
\bar{W}_{-\underline{\alpha}}=\bar{\omega}_{-\underline{\alpha}}+\bar{\eta}^{-}\left[d_{--\underline{\alpha}}+i D_{--} \tau_{\underline{\alpha}}+\frac{1}{2}\left(\Pi \frac{\underline{m}}{--}-\frac{1}{2} \lambda_{-} \Gamma_{\underline{m}} \bar{\lambda}_{-}\right)(\Gamma \underline{m} \theta)_{\underline{\alpha}}\right]-\eta^{-} \bar{\eta}^{-} D_{--} \bar{\omega}_{-\underline{\alpha}},
$$

where $\omega_{-\underline{\alpha}}$ and $\bar{\omega}_{-\underline{\alpha}}$ are the conjugate momenta to $\lambda_{-\underline{\alpha}}$ and $\bar{\lambda}_{-\underline{\alpha}}$ respectively and $\tau_{\underline{\alpha}}$ is the conjugate momentum to the auxiliary field $\sigma_{--}^{\underline{\alpha}}$ of (2.2). The field

$$
d_{--\underline{\alpha}}=p_{--\underline{\alpha}}-\frac{1}{2} \Pi \frac{\underline{m}}{--}\left(\Gamma_{\underline{m}} \theta\right)_{\underline{\alpha}}-\frac{1}{8}\left(D_{--} \theta \Gamma \underline{\underline{m}} \theta\right)\left(\Gamma_{\underline{m}} \theta\right)_{\underline{\alpha}} \approx 0,
$$

which satisfies the Poisson brackets

$$
\left\{d_{\alpha}, d_{\beta}\right\}=-\Gamma \underline{\underline{\underline{\alpha}} \underline{\Pi}} \Pi_{--\underline{m}},
$$

is the usual Green-Schwarz supercovariant momentum.

The component fields of $J_{--}$are given by

$$
\begin{aligned}
j= & \left.J_{--}\right|_{\eta=\bar{\eta}=0}=i\left(\lambda \underline{\underline{\alpha}} \omega_{-\underline{\alpha}}-\bar{\lambda}_{-}^{\underline{\alpha}} \bar{\omega}_{-\underline{\alpha}}\right)+\ldots, \\
G= & -\left.i D_{-} J_{--}\right|_{\eta=\bar{\eta}=0}=\lambda \underline{\underline{\alpha}}\left(d_{--\underline{\alpha}}-i D_{--} \tau_{\underline{\alpha}}\right)+\left(i \sigma_{-}^{\underline{\alpha}}-D_{--} \theta^{\underline{\alpha}}\right) \bar{\omega}_{-\underline{\alpha}}+\ldots, \\
\bar{G}= & \left.i \bar{D}_{-} J_{--}\right|_{\eta=\bar{\eta}=0}=\bar{\lambda}_{-}^{\underline{\alpha}}\left(d_{--\underline{\alpha}}+i D_{--} \tau_{\underline{\alpha}}\right)-\left(i \sigma_{-}^{\underline{\alpha}}+D_{--} \theta^{\underline{\underline{\alpha}}}\right) \omega_{-\underline{\alpha}}+\ldots, \\
T= & \left.\frac{i}{4}\left(D_{-} \bar{D}_{-}-\bar{D}_{-} D_{-}\right) J_{--}\right|_{\eta=\bar{\eta}=0} \\
= & -\omega_{-\underline{\alpha}} D_{--} \lambda \underline{\underline{\alpha}}+\frac{1}{2} D_{--}\left(\lambda_{-}^{\underline{\alpha}} \omega_{-\underline{\alpha}}\right)-\bar{\omega}_{-\underline{\alpha}} D_{--} \bar{\lambda}_{-}^{\underline{\alpha}}+\frac{1}{2} D_{--}\left(\bar{\lambda}_{-}^{\underline{\alpha}} \bar{\omega}_{-\underline{\alpha}}\right) \\
& +D_{--} \theta^{\underline{\alpha}} d_{--\underline{\alpha}}+\sigma_{--}^{\underline{\alpha}} D_{--} \tau_{\underline{\alpha}}-\frac{1}{2} \Pi_{--}^{\underline{m}} \Pi_{--\underline{m}}+\ldots,
\end{aligned}
$$

where '...' denotes terms proportional to the superembedding constraints $(2.10)-(2.13)$.

In the superconformal gauge $\mathcal{E}_{++}^{--}(z)=0, e_{--}^{++}(\xi)=0$, the currents (4.6)-(4.9) generate the right-sector $n=(0,2)$ super Virasoro algebra realized on the matter fields. Thus, in the superconformal gauge, the $n=(0,2)$ superembedding formulation of the heterotic superstring is an interacting $n=(0,2), d=2$ superconformal model. Note that this model is still invariant under the six $\kappa$-symmetries (2.23).

In the next section we shall use these symmetries to gauge fix the superembedding action in such a way that it would transform into the Berkovits action.

\section{Complexified superembedding and Berkovits action}

To quantize the action (3.1) one should gauge fix the worldsheet superdiffeomorphisms and the local $\kappa$-symmetries (2.23). The gauge fixing of the local $\kappa$-symmetries in a way which preserves a $U(4)$ subgroup of the Lorentz group $S O(1,9)$, resulting in a consistent semicovariant quantization of the superstring, has been performed in [8]. However, our present 
goal is to recover from the superembedding the Berkovits pure spinor formulation, which provides one with a $D=10$ super-Poincaré covariant quantization. Therefore, we should proceed in a different way.

The Berkovits action (see eq. (5.16)) does not contain the pure spinor $\bar{\lambda}$. Furthermore, under BRST transformations the 'real' spinor $\theta$ acquires a complex variation proportional to $\lambda$, therefore this action is strictly speaking complex. So we shall also 'complexify' the superembedding model, i.e. consider the superfields (2.1) and (2.2) as complex ones. This procedure makes sense at the quantum level where the fields are operators and the Fock space has in general an indefinite metric.

The complexification has two useful consequences. On the one hand, it allows us to treat $\lambda$ and $\bar{\lambda}$ in an asymmetric fashion and, in particular, to use available local symmetries (2.23) for eliminating the pure spinor $\bar{\lambda}$ from the action (3.1). On the other hand, it allows us to twist the $n=(0,2)$ superconformal model which arises upon gauge fixing the worldsheet superdiffeomorphisms.

Let us begin with expressing $\bar{\lambda}_{-}^{\underline{\alpha}}$ in terms of other fields of the model. To this end let us consider the $U(5)$ subgroup of the (Wick rotated) Lorentz group $S O(10)$, under which an $S O(10)$ vector $V^{\underline{a}} \equiv\left(V^{r}, V_{r}\right), r=1, \ldots, 5$, decomposes as a $\mathbf{5}$-irrep $V^{r}$ and a $\overline{\mathbf{5}}$-irrep $V_{r}=V^{r *}$ of $U(5)$. An $S O(10)$ (chiral) spinor $\phi^{\underline{\alpha}} \equiv\left(\phi^{0}, \phi_{[r s]}, \phi^{r}\right)$ decomposes as $(\mathbf{1}, \overline{\mathbf{1 0}}, \mathbf{5})$ of $U(5)$. Let $v_{\underline{\alpha}}^{0}$ be a constant $1 \times 16 c$-number matrix that extracts from $\phi^{\underline{\alpha}}$ the $U(5)$ singlet $\phi^{0}=v_{\underline{\alpha}}^{0} \phi \underline{\underline{\alpha}}$. Notice that $v_{\underline{\alpha}}^{0}$ satisfies the pure spinor condition $\left(v^{0} \Gamma^{\underline{a}} v^{0}\right)=0 . \bar{\varphi}$ Then one may define the pure spinor

$$
Y_{\underline{\alpha}}^{-}=\frac{v_{\underline{\alpha}}^{0}}{v^{0} D_{-} \Theta}, \quad Y^{-} D_{-} \Theta \equiv 1
$$

and construct the projector

$$
\mathcal{P}^{\underline{\alpha}} \underline{\beta}_{\underline{\beta}}=\frac{1}{2}(\Gamma \underline{m} Y)^{\underline{\alpha}}\left(D_{-} \Theta \Gamma_{\underline{m}}\right)_{\underline{\beta}}, \quad \mathcal{P} \mathcal{P}=\mathcal{P}, \quad(1-\mathcal{P})(1-\mathcal{P})=(1-\mathcal{P}) .
$$

Since $D_{-} \Theta$ is a pure spinor, we have

$$
\mathcal{P} D_{-} \Theta=0, \quad D_{-} \Theta \Gamma^{\underline{m}}(1-\mathcal{P})=0 \text {. }
$$

Note that $\operatorname{Tr} \mathcal{P}=5$ and $\operatorname{Tr}(1-\mathcal{P})=11$, hence $\mathcal{P}$ and $1-\mathcal{P}$ project the 16 -dimensional spinor space onto 5- and 11-dimensional subspaces respectively. In particular, (5.3) reflects the fact that the pure spinor $D_{-} \Theta$ has 11 independent components.

As $Y^{-}$and $\mathcal{P}$ contain $v_{\underline{\alpha}}^{0}$, they are non-covariant, nevertheless they appear only at an intermediate step as a technical tool in the passage from the covariant superembedding to the covariant Berkovits action. In other words, though we shall fix part of the $\kappa$-symmmetries

\footnotetext{
${ }^{8}$ It would be of interest to consider the possibility of promoting the constant $v_{\underline{\alpha}}^{0}$ to a component of a worldsheet harmonic matrix parametrizing a coset space $\frac{S O(10)}{U(5)}$, thus making $v_{\underline{\alpha}}^{0}$ an auxiliary harmonic field (for the use of harmonics in the superembedding description of superstrings see e.g. 31).
} 
(2.23) in a way which breaks $S O(10)$ down to $U(5)$, the complete gauge fixing of all local symmetries will restore the Lorentz covariance.

Consider now the pure spinor condition for $\bar{D}_{-} \Theta$ whose first component is $\bar{\lambda}_{-}$

$$
\bar{D}_{-} \Theta \Gamma^{\underline{m}} \bar{D}_{-} \Theta=0 \text {. }
$$

As it has been discussed, this condition implies that only eleven of the sixteen components of $\bar{D}_{-} \Theta$ are independent. Since any superfield $S^{\underline{\alpha}}$ such that $S=\mathcal{P} S$ identically satisfies the pure spinor condition $S \Gamma \underline{m} S=0$, one can immediately see that the five components of $\bar{D}_{-} \Theta$ 'eliminated' by $(5.4)$ belong to the projected part $(1-\mathcal{P}) \bar{D} \_\Theta$. Also, the six $\kappa^{-}$ transformations $(2.23)$ acting on $\bar{D}_{-} \Theta$ affect only its projected part $(1-\mathcal{P}) \bar{D}_{-} \Theta$, because $\mathcal{P} \delta \Theta \equiv 0$. Since, as we mentioned, $(1-\mathcal{P}) \bar{D}_{-} \Theta$ has eleven independent components, five of which are zero due to (5.4) and the remaining six transform under $\kappa$-symmetry, we can impose the condition

$$
(1-\mathcal{P}) \bar{D}_{-} \Theta=0
$$

which gauge fixes the part of $\kappa$-symmetry (2.23) acting on $\bar{D}_{-} \Theta$. By (2.7) this condition is solved as

$$
\bar{D}_{-} \Theta^{\underline{\alpha}}=\Pi_{--}^{\underline{m}}\left(\Gamma_{\underline{m}} Y^{-}\right)^{\underline{\alpha}},
$$

which for the components of $\bar{D}_{-} \Theta$ implies

$$
\bar{\lambda} \underline{\alpha}=\left.\Pi \frac{\underline{m}}{--}\left(\Gamma_{\underline{m}} Y^{-}\right) \underline{\alpha}\right|_{\eta=\bar{\eta}=0},
$$

and (due to $(5.3))$

$$
\left.\left(D_{--} \theta-i \sigma_{--}\right)^{\underline{\alpha}}=2 \mathcal{P}^{\underline{\alpha}}{ }_{\beta} D_{--} \theta \underline{\beta}+D_{--}\left(\chi_{-}^{\underline{m}}-\theta \Gamma^{\underline{m}} \lambda_{-}\right)\left(\Gamma_{\underline{m}} Y^{-}\right) \underline{\alpha}\right),
$$

or taking into account 2.10$)$

$$
\sigma_{--}^{\underline{\alpha}}=-i\left[(1-2 \mathcal{P}) D_{--} \theta\right]^{\underline{\alpha}} .
$$

In (5.8) and (5.9) $\mathcal{P}$ and $Y^{-}$stand for the leading components of the superfields (5.2) and (5.1), that below we shall denote with the same symbol. We thus expressed $\bar{\lambda}_{-}^{\underline{\alpha}}$ and $\sigma_{--}^{\underline{\alpha}}$ in terms of $\lambda^{\underline{\alpha}}$ and (the derivatives of) $\theta \underline{\alpha}$ and $x \underline{\underline{m}}$.

Note that the gauge fixing condition (5.7) and the constraint $\bar{\omega}_{-\underline{\alpha}} \approx 0$ on the momentum $\bar{\omega}_{-\underline{\alpha}}$, conjugate to $\bar{\lambda} \frac{\alpha}{-}$, introduced in $(4.3)$, form a canonical pair of second class constraints under the Poisson brackets, i.e. $\left[\bar{\lambda}_{-}^{\underline{\alpha}}-\Pi_{--}^{\underline{m}}\left(\Gamma_{\underline{m}} Y^{-}\right) \underline{\alpha}, \bar{\omega}_{-\underline{\beta}}\right]=\delta_{\underline{\beta}}$. It follows that $\bar{\omega}_{-\underline{\alpha}}$ can be considered to vanish in the strong sense and therefore can be dropped in the definition of the $n=(0,2)$ supercurrent components (4.6)-(4.9). The same reasoning applies to $\sigma_{-}^{\underline{\alpha}}$ - and its conjugate momentum $\tau_{\underline{\alpha}}$ which can be strongly put to zero.

The expressions (5.7) and (5.9) identically satisfy the superembedding conditions (2.13). Then, substituting (5.7) into the twistor-like constraint (2.12), we reduce it to

$$
\frac{1}{2} Y^{-} \Gamma^{\underline{m}} \Gamma_{\underline{n}} \lambda_{-} \Pi_{--}^{\underline{n}}=0 .
$$


Apart from the pure spinor condition $\lambda_{-} \Gamma^{\underline{m}} \lambda_{-}=0$, which we further assume to be satisfied in the strong sense. If this is the only constraint which remains in the model. So, upon substituting the expression for $\bar{\lambda}_{-}$(5.7) into the action (3.1), we have

$$
\begin{aligned}
I_{S E} & =\frac{1}{2} \int d^{2} \xi\left[\Pi \frac{m}{++} \Pi_{--\underline{m}}+\frac{1}{2} \Pi \frac{m}{++} D_{--} \theta \Gamma_{\underline{m}} \theta-\frac{1}{2} \Pi \underline{\underline{m}} D_{++} \theta \Gamma_{\underline{m}} \theta\right. \\
& \left.+\bar{\psi}_{+}^{I} D_{--} \psi_{+}^{I}+\psi_{+}^{I} D_{--} \bar{\psi}_{+}^{I}\right]+\frac{1}{2} \int d^{2} \xi p_{++\underline{m}}\left(Y \Gamma \underline{\underline{m}} \Gamma_{\underline{n}} \lambda \Pi_{--}^{\underline{n}}\right)
\end{aligned}
$$

or a la Siegel [33

$$
\begin{aligned}
I_{S E} & =\int d^{2} \xi\left[\frac{1}{2} \partial_{++} x \underline{\underline{m}} D_{--} x_{\underline{m}}+p_{--} \partial_{++} \theta-d_{--} \partial_{++} \theta+\bar{\psi}_{+}^{I} D_{--} \psi_{+}^{I}+\psi_{+}^{I} D_{--} \bar{\psi}_{+}^{I}\right] \\
& +\frac{1}{2} \int d^{2} \xi p_{++\underline{m}}\left(Y \Gamma^{\underline{m}} \Gamma_{\underline{n}} \lambda \Pi_{--}^{\underline{n}}\right) .
\end{aligned}
$$

The action (5.11), and therefore also (5.12), is invariant under the $n=(0,2)$ superdiffeomorphisms and under the following $\kappa$-symmetry transformations (2.23) remained upon gauge fixing $\bar{\lambda}_{-}$and $\sigma_{--}$

$$
\begin{aligned}
\delta \theta^{\underline{\alpha}} & =\left(\delta_{\underline{\beta}}^{\underline{\alpha}}-\lambda \underline{\underline{\alpha}} Y_{\underline{\beta}}\right) \Gamma \frac{\beta \gamma}{\underline{n}} \tilde{\kappa}_{\underline{\gamma}}^{--}\left(\lambda \Gamma^{\underline{n}} \Gamma_{\underline{m}} Y\right) \Pi_{--}^{\underline{m}}, \\
\delta \lambda^{\underline{\alpha}} & =\left(\delta \underline{\underline{\beta}}-\lambda \underline{\alpha} Y_{\underline{\beta}}\right) \Gamma \frac{\beta \gamma}{\underline{\underline{\gamma}}}\left(\lambda \Gamma^{\underline{n}} \Gamma_{\underline{m}} Y\right)\left[\Pi \underline{-}-\tilde{\mu}_{\underline{\gamma}}^{-}-\left(\lambda_{-} \Gamma^{\underline{m}} D_{--} \theta\right) \tilde{\kappa}_{\underline{\underline{\gamma}}}^{--}\right], \\
\delta p_{++\underline{m}} & =-\delta \Pi_{++\underline{m}}=\partial_{++} \theta \Gamma_{\underline{m}} \delta \theta
\end{aligned}
$$

where $\tilde{\kappa}_{\underline{\gamma}}^{--}=\kappa_{\underline{\gamma}}^{--}-Y_{\underline{\gamma}}\left(\lambda \kappa^{--}\right)$and $\tilde{\mu}_{\underline{\gamma}}^{-}=\left.\left(\delta \underline{\underline{\alpha}}-Y_{\underline{\gamma}} \lambda^{\underline{\alpha}}\right) D_{-} K_{\underline{\alpha}}^{--}\right|_{\eta=\bar{\eta}=0}$ are components of the superfield $\kappa$-symmetry parameter $(\overline{2.23})$.

Let us now compare (5.12) with the Berkovits action

$$
I_{B}=\int d^{2} \xi\left[\frac{1}{2} \partial_{++} x^{\underline{m}} \partial_{--} x_{\underline{m}}+p_{--} \partial_{++} \theta+\omega_{--} \partial_{++} \lambda+\bar{\psi}_{+}^{I} \partial_{--} \psi_{+}^{I}+\psi_{+}^{I} \partial_{--} \bar{\psi}_{+}^{I}\right] .
$$

In this action, which describes a free conformal system, $\lambda$ and $\omega_{--}$have conformal weights 0 and 1 , and ghost numbers 1 and -1 , respectively, while in (5.12) $\lambda_{-}$, as well as its conjugate momentum $\omega_{-}$, has conformal weight $\frac{1}{2}$ and ghost number 0 .

Therefore, as a first step in establishing the relationship between the actions (5.12) and (5.16), we should change the conformal weights of $\lambda_{-}$and $\omega_{-}$and endow them with appropriate ghost numbers. The complexification allows one to do this via 'twisting'. Indeed, before the complexification the complex conjugate Grassmann coordinates $\eta^{-}$and $\bar{\eta}^{-}$must have the same weight fixed to be $-\frac{1}{2}$ because of the relation $D_{-} \bar{D}_{-}+\bar{D}_{-} D_{-}=2 D_{--}$. However, after the complexification $\eta$ and $\bar{\eta}$ become independent variables, and one can

\footnotetext{
${ }^{9}$ In a variation of the pure spinor quantization method considered in 32] the pure spinor constraint has been handled as a relaxed condition.
} 
choose their weights to be respectively $-\frac{1}{2}+w_{0}$ and $-\frac{1}{2}-w_{0}$, with arbitrary $w_{0}$. Choosing $w_{0}=\frac{1}{2}$ one sees, by eqs. (2.2) and (4.3), that $\lambda$ acquires weight 0 and $\omega$ weight 1 . Similarly, the supersymmetry ghosts $\gamma_{-}$and $\bar{\gamma}_{-}(2.21)$ change their weights from $-\frac{1}{2}$ and $-\frac{1}{2}$ to 0 and -1 , respectively. As far as the change of the ghost number is concerned, to turn the 'matter' fields $\omega_{-}$and $\lambda_{-}$into the ghost system with conformal weights $(1,0)$ and ghost numbers $(-1,1)$, one can use the ghost field $\gamma^{-}$and make the field redefinition $\lambda=\gamma^{-} \lambda_{-}$ and $\omega_{--}=\frac{1}{\gamma^{-}} \omega_{-}$. Actually, this can be done with the same result before or after the twist.

In more precise terms the twisting procedure consists in the following. One should first gauge fix the $n=(0,2)$ superdiffeomorphisms (2.20) of the action (5.12) to reduce it to an $n=(0,2)$ superconformal action. To this end, we impose as gauge fixing that both the Beltrami parameter corresponding to the left-sector diffeomorphisms and the one corresponding to the right-sector superdiffeomorphisms vanish, i.e.

$$
e_{--}^{++}=0, \quad \mathcal{E}_{++}^{--}=0
$$

This gauge fixing requires the introduction of the system of superdiffeomorphism ghosts (2.21) and (2.22).

In what follows we shall not consider the left sector diffeomorphism ghosts, whose treatment is assumed to follow the standard BRST procedure for the bosonic string, and will concentrate on the right-moving (supersymmetric) sector of the model whose quantization is problematic.

The system of the right-sector $n=(0,2)$ superdiffeomorphism ghosts (2.21) and (2.22) consists of the fermionic ghost pairs $\left(b, c^{--}\right)$of weights $(2,-1)$ and $(u, v)$ of weights $(1,0)$, associated, respectively, with the right-sector bosonic diffeomorphisms and the $U(1) \mathrm{R}-$ symmetry, and of bosonic ghost pairs $\left(\beta, \gamma^{-}\right)$and $\left(\bar{\beta}, \bar{\gamma}^{-}\right)$of weights $\left(\frac{3}{2},-\frac{1}{2}\right)$ associated with the local $n=(0,2)$ supersymmetries. The $n=(0,2)$ ghost currents

$$
\begin{aligned}
j_{g h} & =i(\beta \gamma-\bar{\beta} \bar{\gamma}) \\
G_{g h} & =-i \beta(v+i \partial c)-i \bar{\gamma}(b+i \partial u), \\
\bar{G}_{g h} & =i(b-i \partial u) \gamma+i \bar{\beta}(v-i \partial c), \\
T_{g h} & =2[i(\partial \beta) \gamma+i \bar{\beta} \partial \bar{\gamma}+(\partial c) b+(\partial u) v],
\end{aligned}
$$

should be added to the matter currents (4.6)-(4.9) which, upon the elimination of $e_{--}^{++}, \bar{\lambda}_{-}$, $\sigma_{--}$and their momenta, take the form

$$
\begin{aligned}
j & =i \lambda \underline{\underline{\alpha}} \omega_{-\underline{\alpha}}, \\
G & =\lambda \underline{-} d_{--\underline{\alpha}}, \\
\bar{G} & =\Pi \underline{\underline{m}} Y^{-} \Gamma_{\underline{m}} d_{--}-2 \omega_{-}(1-\mathcal{P}) \partial_{--} \theta \\
T & =-\omega_{-\underline{\alpha}} \partial_{--} \lambda_{-}^{\underline{\alpha}}+\frac{1}{2} \partial_{--}\left(\lambda \underline{\alpha} \omega_{-\underline{\alpha}}\right)+\partial_{--} \theta^{\underline{\alpha}} d_{--\underline{\alpha}}-\frac{1}{2} \Pi_{--}^{\underline{m}} \Pi_{--\underline{m}} .
\end{aligned}
$$


The currents $j+j_{g h}, G+G_{g h}, \bar{G}+\bar{G}_{g h}$ and $T+T_{g h}$ form the $n=(0,2)$ superconformal algebra of the matter + ghost system.

The twist of the $n=2$ superconformal algebra [30] consists in shifting the stress-energy tensor $T$ as follows

$$
T \rightarrow T^{\prime}=T+\frac{i}{2} \partial j
$$

This corresponds to adding a charge at infinity and has two important consequences:

i) the conformal weight $w$ of any field $\phi$ with $R$-charge $q$ gets shifted

$$
w \rightarrow w^{\prime}=w-\frac{1}{2} q
$$

ii) whatever the central charge was, it vanishes after the twist.

For instance, after the twist the conformal weights of the pairs $\left(\beta, \gamma^{-}\right)$and $\left(\bar{\beta}, \bar{\gamma}^{-}\right)$ become $(1,0)$ and $(2,-1)$ respectively, and the central charge of the ghost part of the $n=$ $(0,2)$ superconformal generators, which was -6 , vanishes after the twist. Because of this, and since the ghost sector is completely decoupled from the matter fields, we can exclude the superdiffeomorphim ghosts from the consideration and neglect them hereafter. This is the reason why the superdiffeomorphism ghosts and, in particular, the $(b, c)$ system, are not present in the pure spinor formalism.

We are thus left with the 'matter' part of the $n=(0,2)$ superconformal system, in which the conformal weights get shifted from $\left(\frac{1}{2}, \frac{1}{2}\right)$ to $(1,0)$, i.e. $\left(\omega_{-}, \lambda_{-}\right) \rightarrow\left(\omega_{--}, \lambda\right)$.

Then, according to [30], after the twist one can reinterpret $i j$ as the ghost number current with conformal weight one and zero ghost number. Thus $\lambda$ acquires the ghost number one and $\omega_{--}$becomes a field with $n_{g h}=-1$. To As a consequence, $G=\lambda d_{--}$becomes a conserved current with $w=1$ and $n_{g h}=1$ and can be identified with a BRST current whose associated BRST charge

$$
Q_{B}=\oint \lambda d_{--}
$$

is nilpotent, since $G G=0$ in virtue of the pure spinor property of $\lambda . \bar{G}$ acquires conformal weight 2 and ghost number -1 and is therefore interpreted as the composite antighost field

$$
b_{B}=\frac{1}{2} \bar{G}=\frac{1}{2} \Pi \underline{\underline{m}} Y \Gamma_{\underline{m}} d_{--}-\omega_{--}(1-\mathcal{P}) \partial_{--} \theta .
$$

We have thus shown that the twisting of the $n=(0,2)$ superconformal algebra reproduces the BRST current and the antighost field $b_{B}$ of the Berkovits pure spinor formulation [25]. In

\footnotetext{
${ }^{10}$ This is essentially the same as to say that one has made the field redefinition of $\lambda$ and $\omega$ with the use of the ghost $\gamma$.
} 
particular, the antighost $b_{B}$, which is required for the construction of higher genus amplitudes, satisfies the Poisson bracket anticommutation relation

$$
\left\{Q_{B}, b_{B}(\xi)\right\}=2 T^{\prime}(\xi)
$$

Though $b_{B}$ is not Lorentz invariant, a consequence of the non-invariance of $Y$ and $\mathcal{P}$, its variation under the constant Lorentz transformations $\Lambda_{\underline{m n}}$ is BRST exact

$$
\delta b_{B}=\frac{1}{2}\left\{Q_{B}, \Pi_{--}^{\underline{m}} \omega_{--}(1-\mathcal{P}) \Gamma_{\underline{m}} \delta Y\right\}+\frac{1}{16} \Lambda_{\underline{m n}}\left\{Q_{B},\left(Y \Gamma^{\underline{m}} d_{--}\right)\left(Y \Gamma^{\underline{n}} d_{--}\right)\right\},
$$

where $\delta Y_{\underline{\alpha}}=\frac{1}{4} \Lambda_{\underline{m n}}(Y \Gamma \underline{m n}) \underline{\beta}\left(\delta_{\underline{\alpha}}^{\frac{\beta}{\alpha}}-\lambda^{\beta} Y_{\underline{\alpha}}\right)$. Eqs. (5.30) and (5.31) point to the fact that the physical amplitudes should remain Lorentz covariant despite the non-invariance of $b_{B}$.

In view of the above reasoning we identify $Q_{B}$ with the BRST charge of the quantized theory and use it to gauge fix the remaining $\kappa$-symmetries (5.13) of the action (5.12). To this end we introduce the gauge fermion

$$
\mathcal{F}=\mathcal{F}_{1}+\mathcal{F}_{2}=\frac{1}{2} p_{++\underline{m}} d_{--} \Gamma^{\underline{m}} Y+\omega_{--}(1-\mathcal{P}) \partial_{++} \theta,
$$

and add to the action (5.12) the gauge fixing term

$$
\begin{aligned}
I_{g f} & =\int d^{2} \xi\left\{Q_{B}, \mathcal{F}\right\} \\
& =\int d^{2} \xi\left[-\frac{1}{2} p_{++\underline{m}}\left(Y \Gamma \underline{\underline{m}} \Gamma_{\underline{n}} \lambda \Pi_{--}^{\underline{n}}\right)+d_{--} \mathcal{P} \partial_{++} \theta+d_{--}(1-\mathcal{P}) \partial_{++} \theta+\omega_{--} \partial_{++} \lambda\right],
\end{aligned}
$$

where we have used the BRST variations

$$
\begin{gathered}
\left\{Q_{B}, p_{++\underline{m}}\right\}=-\partial_{++} \theta \Gamma_{\underline{m}} \lambda, \quad\left\{Q_{B}, d_{--}\right\}=-\lambda \Gamma_{\underline{n}} \Pi \underline{\underline{n}}, \\
\left\{Q_{B}, \omega_{--}\right\}=d_{--}, \quad\left\{Q_{B}, \theta\right\}=\lambda .
\end{gathered}
$$

The resulting action $I_{S E}+I_{g f}$ is just the Lorentz covariant Berkovits action (5.16). The first term in (5.33) cancels the residual superembedding term of (5.12), the second and third terms cancel $d_{--} \partial_{++} \theta$ and the last one reproduces the kinetic contribution to the pure spinor ghost $\lambda$.

Note that in 29] the second term of (5.33) has been added to the Green-Schwarz action 'by hand' to render it invariant under the BRST transformation generated by $Q_{B}$, and the gauge fermion introduced therein was the $\mathcal{F}_{2}$ part of (5.32). In the superembedding formulation all the required terms naturally appear in the BRST-exact gauge fixing part of the superstring action.

We have thus shown that the Berkovits action is the gauge fixed action of the complexified and twisted $n=(0,2)$ superembedding formulation of the superstring. Therefore, since 
the latter is classically equivalent to the Green-Schwarz formulation, we have established the relationship between the Green-Schwarz and the pure spinor superstring action.

An interesting problem, which should yet be understood, is the relation between the Berkovits BRST charge (5.28), which appears at the stage of twisting, and a conventional BRST charge which one should construct when trying to quantize the superembedding action (5.11) directly. The conventional BRST charge of the model should include all the constraints generating the $n=(0,2)$ superconformal algebra (5.22) and the $\kappa$-symmetry transformations (5.13) and has the form

$$
Q_{B R S T}=\oint\left[\left(\gamma^{-} \lambda_{-}\right) d_{--}+\bar{\gamma} \bar{G}+v j+c T+c^{\kappa} T_{\kappa}+O(3)\right],
$$

where the first term in (5.34) is just the Berkovits BRST charge with $\lambda=\gamma^{-} \lambda_{-}, T_{\kappa}$ are $\kappa$-symmetry generators with $c^{\kappa}$ being the corresponding ghosts, and $O(3)$ stands for cubic (anti)ghost terms.

It is known that, for example, in the case of the Berkovits-Vafa embedding of a bosonic string into an $n=1$ fermionic string, and an $n=1$ fermionic string into an $n=2$ fermionic string [30], there is a similarity transformation involving the BRST charges of these theories [34] (see also [35] for higher $n$ ), namely

$$
e^{-R} Q_{n+1} e^{R}=Q_{n}+Q_{t o p},
$$

where $Q_{t o p}$ is the BRST charge of a topological sector of trivial cohomology.

It would be of interest to understand whether an analogous transformation exists that relates the BRST charge (5.34) with the Berkovits charge (5.28). For studying this point it might be useful to implement the observation of McArthur [36] that the transformation (5.35) is related to a non-linear realization of the symmetry associated with $Q_{n+1}$.

\section{Generalization to a supergravity-SYM background}

Using results of [29], we can generalize the above consideration to the superembedding action (2.15) which describes the heterotic string propagating in a curved background which satisfies the constraints of $N=1, D=10$ supergravity interacting with $N=1, D=10$ super-YangMills. The detailed analysis of the $N=1, D=10$ supergravity-SYM constraints relevant to the consideration below may be found in 37. Upon complexification and the gauge fixing of the pullback of the spinor supervielbein $\bar{E}_{-}^{\underline{\alpha}}(2.4)$, which replaces $\bar{D}_{-} \Theta$ and $\bar{\lambda}_{-}$of the flat case, we get the generalization of the component action (3.1) to the curved superbackground in the following form

$$
\begin{aligned}
I_{S E} & =\frac{1}{2} \int d^{2} \xi\left[E_{++}^{\underline{a}} E_{--\underline{a}}+B_{++,--}+\bar{\psi}_{+}\left(D_{--}+A_{--}\right) \psi_{+}+\psi_{+}\left(D_{--}+A_{--}\right) \bar{\psi}_{+}\right] \\
& +\frac{1}{2} \int d^{2} \xi p_{++\underline{a}}\left(Y^{-} \Gamma^{\underline{a}} \Gamma_{\underline{b}} \lambda_{-} E_{--}^{\underline{b}}\right)
\end{aligned}
$$


where now $\lambda_{-}^{\underline{\alpha}}=E_{-}^{\underline{\alpha}}$, and $E_{ \pm \pm}^{\underline{A}}, E_{-}^{\underline{\alpha}}$ and $B_{++,--}$stand for the leading $(\eta=\bar{\eta}=0)$ components of the pullbacks of the supervielbein (2.4) and of the NS-NS two-form $B^{(2)}$. $A_{--}^{I J}=\partial_{--} Z^{\underline{M}} A_{\underline{M}}^{I J}$ is the pullback of the super-Yang-Mills potential.

The variations of $\lambda \stackrel{\underline{\alpha}}{-}$, the Lagrange multiplier $p_{++\underline{a}}$ and the heterotic fermions $\psi_{+}^{I}$ under the $n=(0,2)$ supersymmetry and $\kappa$-symmetry transformations (2.23) are now

$$
\begin{gathered}
\delta \lambda_{-}^{\underline{\alpha}}=\delta Z^{\underline{M}} \Omega_{\underline{M} \underline{\underline{\beta}}} \lambda_{-}^{\underline{\alpha}}, \\
\delta p_{++\underline{a}}=\left(E_{++}+W^{I J} \bar{\psi}_{+}^{I} \psi_{+}^{J}\right) \Gamma_{\underline{a}} \delta Z^{\underline{M}} E_{\underline{M}}-\frac{1}{2} p_{++\underline{b}} \delta Y \Gamma^{\underline{b}} \Gamma_{\underline{a}} \lambda, \\
\delta \psi_{+}^{I}=-\delta Z^{\underline{M}} E_{\underline{\underline{M}}}^{\underline{\alpha}} A_{\underline{\alpha}}^{I J} \psi_{+}^{J},
\end{gathered}
$$

where $\Omega_{++\underline{\underline{\alpha}}} \underline{\underline{\beta}}=d Z \underline{\underline{M}} \Omega_{\underline{M \alpha}} \underline{\underline{\beta}}=d \Phi \delta_{\underline{\alpha}}^{\underline{\beta}}+\Omega^{\underline{a b}} \Gamma_{\underline{a b \alpha}} \underline{\underline{\beta}}$ is a spin connection containing the differential of the dilaton superfield $\Phi(Z)$ and the $S O(1,9)$ spin connection, $W^{\underline{\alpha} I J}=\Gamma \underline{a \alpha \underline{\beta}} F_{\underline{a} \beta}^{I J}$ and $F^{I J}=(d A+A \wedge A)^{I J}$ is a constrained super-Yang-Mills stress tensor.

Note that the $n=(0,2)$ SUSY variation (6.2) of $\lambda \underline{-}$ induces local $S O(1,9)$ Lorentz rotations with the parameter $\delta Z^{\underline{M}} \Omega_{\underline{\underline{a}} \underline{\underline{b}}} \Gamma_{\underline{a} \underline{b}}$. This Lorentz transformation could be compensated in (6.1) by the corresponding Lorentz rotations of $p_{++\underline{a}}, E_{-}^{\underline{b}}$ and $Y_{\underline{\alpha}}^{-}$. But by construction (5.1) $Y_{\underline{\alpha}}^{-}$is not Lorentz covariant, the 'anomalous' Lorentz variation of $Y_{\underline{\alpha}}^{-}$being

$$
\delta Y_{\underline{\alpha}}=\delta Z^{\underline{M}}\left(\Omega_{\underline{M} \underline{\alpha}}^{\underline{\beta}} Y_{\underline{\beta}}-Y_{\underline{\alpha}} \Omega_{\underline{M} \underline{\underline{\gamma}}}^{\underline{\beta}} Y_{\underline{\beta}} \lambda \underline{\gamma}\right), \quad \lambda \delta Y \equiv 0 .
$$

In order to cancel this variation of $Y_{\underline{\alpha}}$ the variation of the Lagrange multiplier $p_{++\underline{a}}$ should acquire the last term (6.3).

We now twist and gauge fix the model as in Section 5 with the gauge fermion

$$
\mathcal{F}=p_{++\underline{a}} d_{--} \Gamma^{\underline{a}} Y+\omega_{--}(1-\mathcal{P})\left(E_{++}+W^{I J} \bar{\psi}_{+}^{I} \psi_{+}^{J}\right)+\frac{1}{2} p_{++\underline{a}} \omega_{--}(1-\mathcal{P}) \Gamma^{\underline{a}}\left\{Q_{B}, Y\right\},
$$

the resulting action $I_{S E}+\int d^{2} \xi\left\{Q_{B}, \mathcal{F}\right\}$ is the Berkovits action in the $N=1, D=10$ supergravity and super-Yang-Mills background [29, 37]

$$
\begin{aligned}
I_{B}= & \frac{1}{2} \int d^{2} \xi\left[E_{++}^{\underline{a}} E_{--\underline{a}}+B_{++,--}+\bar{\psi}_{+}\left(\partial_{--}+A_{--}\right) \psi_{+}+\psi_{+}\left(\partial_{--}+A_{--}\right) \bar{\psi}_{+}\right] \\
& +\int d^{2} \xi\left[d_{--\underline{\alpha}}\left(E_{++}^{\underline{\alpha}}+W^{\underline{\alpha} I J} \bar{\psi}_{+}^{I} \psi_{+}^{J}\right)+\omega_{--}\left(\partial_{++}+\Omega_{++}+\frac{1}{2} U^{I J} \bar{\psi}_{+}^{I} \psi_{+}^{J}\right) \lambda\right],
\end{aligned}
$$

where $\Omega_{++\underline{\alpha}}^{\underline{\beta}}=\partial_{++} Z^{\underline{M}} \Omega_{\underline{M} \underline{\alpha}}$ is a spin connection, $U_{\underline{\alpha}}^{I J \underline{\beta}}=\nabla_{\underline{\alpha}} W^{I J \underline{\beta}}$ and $\nabla_{\underline{\alpha}}=E \underline{\underline{\mu}}\left[\partial_{\underline{M}}+\Omega_{\underline{M}}+\right.$ $\left.A_{\underline{M}}\right]$ is a target superspace covariant spinor derivative (see [37] for details). Note that in (6.7) we used the following BRST relations

$$
\left\{Q_{B}, Y_{\underline{\alpha}}\right\}=\lambda \underline{\underline{\delta}}\left(\Omega_{\underline{\delta \alpha}}^{\underline{\beta}} Y_{\underline{\beta}}-Y_{\underline{\alpha}} \Omega_{\underline{\delta} \underline{\gamma}}^{\underline{\beta}} Y_{\underline{\beta}} \lambda{ }^{\gamma}\right),
$$




$$
\begin{gathered}
\left\{Q_{B}, p_{++\underline{\alpha}}\right\}=-\left(E_{++}+W^{I J} \bar{\psi}_{+}^{I} \psi_{+}^{J}\right) \Gamma_{\underline{a}} \lambda-\frac{1}{2} p_{++\underline{b}}\left\{Q_{B}, Y\right\} \Gamma^{\underline{b}} \Gamma_{\underline{a}} \lambda, \\
\left\{Q_{B}, d_{--}\right\}=-\lambda \Gamma_{\underline{a}} E_{-}^{\underline{a}}, \\
\left\{Q_{B}, E_{++}^{\underline{\alpha}}\right\}=\left[\left(\partial_{++}+\Omega_{++}\right) \lambda\right]^{\underline{\alpha}}, \quad\left\{Q_{B}, W^{I J} \bar{\psi}_{+}^{I} \psi_{+}^{J}\right\}=\frac{1}{2} \lambda U^{I J} \bar{\psi}_{+}^{I} \psi_{+}^{J} .
\end{gathered}
$$

This concludes the reconstruction of the link between the superembedding and the pure spinor formulation of the superstring.

\section{Conclusion}

We have obtained the pure spinor BRST charge, the antighost, and the corresponding action for the heterotic string introduced by Berkovits by gauge-fixing and twisting the $n=(0,2)$, $d=2$ superdiffeomorphism invariant heterotic string action of the geometrical superembedding formulation. Since the superembedding is classically equivalent to the Green-Schwarz formulation, we have thus related, via superembedding, the Green-Schwarz and the pure spinor superstring action.

As a natural generalization of these results one may wonder how to demonstrate the analogous relation for the type II $D=10$ superstrings and the $D=11$ supermembrane. To this end one should know the $n=2$ superdiffeomorphism invariant form of superembedding actions for these objects. By now only an $n=(1,1), d=2$ worldsheet superfield action for a type IIB superstring [38 and an $n=1, d=3$ worldvolume superfield action for a supermembrane [39] (see also [40]) have been constructed. The $n=(1,1), d=2$ formulation of the type IIA superstring can be obtained from the supermembrane action by the doubledimensional reduction. The problem is to promote these actions to be $n=(2,2), d=2$ and $n=2, d=3$ supersymmetric.

The Berkovits quantization method has given a recipe of how to compute, in a manifestly super-Poincaré covariant manner, tree-level amplitudes of quantum superstring states. This is useful for the analysis of the quantum superstring theory in super-Yang-Mills and supergravity backgrounds, especially with nonzero Ramond-Ramond fields, and for the derivation of the corresponding effective field theories. An important problem for applications of the pure spinor quantization method is to understand a systematic way of constructing one- and higher-loop amplitudes. As we have already mentioned this should involve the composite antighost field (5.29) and requires an additional insight into the proof of Lorentz-invariance of the higher-loop amplitudes.

\section{Acknowledgements}

It is a pleasure to thank N. Berkovits for interest in this work and for illuminating discussions on his formulation of superstrings. We are also grateful to I. Bandos, P. Budinich, F. Fucito, 
P. A. Grassi, P. Pasti, I. Pesando, G. Policastro and K. Skenderis for useful discussions. This work was partially supported by the European Community's Human Potential Programme under contract HPRN-CT-2000-00131 Quantum Spacetime (L.M., M.M., D.S. and M.T.), by the Grant N 383 of the Ukrainian State Fund for Fundamental Research (D.S.) by the INTAS Research Project N 2000-254 (M.M., D.S., and M.T.) and by the Grant N 14540277 from the Japan Society for the Promotion of Science (I.O.).

\section{References}

[1] D. Sorokin, Superbranes and Superembeddings, Phys. Rep. 329 (2000) 1, arXiv:hepth/9906142]; Introduction to the Superembedding Description of Superbranes, arXiv:hep-th/0105102.

[2] J. A. de Azcarraga and J. Lukierski, Supersymmetric Particles with Internal Symmetries and Central Charges, Phys. Lett. B113 (1982) 170; Supersymmetric Particles in N=2 Superspace: Phase Space Variables and Hamiltonian Dynamics, Phys. Rev. D 28 (1983) 1337.

W. Siegel, Hidden Local Supersymmetry in the Supersymmetric Particle Action, Phys. Lett. B128 (1983) 397.

[3] D. P. Sorokin, V. I. Tkach and D. V. Volkov, Superparticles, Twistors and Siegel Symmetry, Mod. Phys. Lett. A4 (1989) 901.

D. P. Sorokin, V. I. Tkach, D. V. Volkov and A. A. Zheltukhin, From the Superparticle Siegel Symmetry to the Spinning Particle Proper Time Supersymmetry, Phys. Lett. B216 (1989) 302.

[4] A. Galperin and E. Sokatchev, A Twistor Like D $=10$ Superparticle Action with Manifest N=8 Worldline Supersymmetry, Phys. Rev. D 46 (1992) 714, arXiv:hepth/9203051].

[5] N. Berkovits, A Covariant Action for the Heterotic Superstring with Manifest SpaceTime Supersymmetry and World Sheet Superconformal Invariance, Phys. Lett. B232 (1989) 184.

[6] M. Tonin, World Sheet Supersymmetric Formulations of Green-Schwarz Superstrings, Phys. Lett. B266 (1991) 312; Kappa Symmetry as World Sheet Supersymmetry in D = 10 Heterotic Superstring, Int. J. Mod. Phys. A7 (1992) 6013.

[7] N. Berkovits, Twistors, N=8 Superconformal Invariance and the Green-Schwarz Superstring, Nucl. Phys. B358 (1991) 169. 
[8] N. Berkovits, The Heterotic Green-Schwarz Superstring on an $N=(2,0)$ Superworldsheet, Nucl. Phys. B379 (1992) 96, arXiv:hep-th/9201004.

[9] F. Delduc, E. Ivanov and E. Sokatchev, Twistor Like Superstrings with $D=3, D=4$, $D=6$ Target Superspace and $N=(1,0), N=(2,0), N=(4,0)$ World Sheet Supersymmetry, Nucl. Phys. B 384 (1992) 334 [arXiv:hep-th/9204071].

F. Delduc, A. Galperin, P. S. Howe and E. Sokatchev, A Twistor Formulation of the Heterotic D $=10$ Superstring with Manifest (8,0) World Sheet Supersymmetry, Phys. Rev. D 47 (1993) 578, arXiv:hep-th/9207050].

[10] I. Bandos, Superembedding Approach and S-Duality: A Unified Description of Superstring and Super-D1-Brane, Nucl. Phys. B 599 (2001) 197 [arXiv:hep-th/0008249].

[11] P. Pasti and M. Tonin, Twistor Like Formulation of the Supermembrane in D $=11$, Nucl. Phys. B418 (1994) 337, arXiv:hep-th/9303156.

E. Bergshoeff and E. Sezgin, Twistor-like Formulation of Super p-Branes, Nucl. Phys. B422 (1994) 329, arXiv:hep-th/9312168.

[12] I. A. Bandos, D. P. Sorokin, M. Tonin, P. Pasti and D. V. Volkov, Superstrings and Supermembranes in the Doubly Supersymmetric Geometrical Approach, Nucl. Phys. B446 (1995) 79, arXiv:hep-th/9501113.

[13] P. S. Howe and E. Sezgin, Superbranes, Phys. Lett. B390 (1997) 133, arXiv:hep th/9607227; $D=11, p=5$, Phys. Lett. B394 (1997) 62, arXiv:hep-th/9611008.

[14] S. J. Gates and H. Nishino, D =2 Superfield Supergravity, Local (Supersymmetry)**2 and Nonlinear Sigma Models, Class. Quant. Grav. 3 (1986) 391.

R. Brooks, F. Muhammed and S. J. Gates Jr., Matter Coupled to D = 2 Simple Unidexterous Supergravity, Local (Supersymmetry)**2 and Strings, Class. Quantum Grav. 3 (1986) 745.

J. Kowalski-Glikman, Doubly Graded Sigma Model with Torsion, Phys. Lett. B180 (1986) 358.

J. Kowalski-Glikman, J. W. van Holten, S. Aoyama and J. Lukierski, The Spinning Superparticle, Phys. Lett. B201 (1988) 487.

A. Kavalov and R.L. Mkrtchyan, Spinning Superparticles, Preprint Yer.PhI 1068(31)88, Yerevan, 1988 (unpublished).

J. M. Fisch, The N=1 Spinning Superstring: A Model of the Super Superstring, Phys. Lett. B219 (1989) 71.

[15] E. Cartan, Lecons sur la Theorie des Spineurs, Hermann, Paris, 1937.

C. Chevalley, The Algebraic Theory of Spinors, Columbia U.P., New York, 1954. 
[16] P. Budinich, From the Geometry of Pure Spinors with their Division Algebras to Fermion's Physics, arXiv:hep-th/0107158.

P. Budinich and A. Trautman, Fock Space Description of Simple Spinors, J. Math. Phys. 30 (1989) 2125.

[17] M. Tonin, Twistor-like Formulation of Heterotic Strings, At X Italian conference on general relativity and gravitational physics - Bardonecchia, September 1 - 5 (1992), in "Bardonecchia 1992, General relativity and gravitational physics", 435-452, arXiv:hepth/9301055].

[18] B.E.W. Nilsson, Pure Spinors as Auxiliary Fields in the Ten-dimensional Supersymmetric Yang-Mills Theory, Class. Quant. Grav. 3 (1986) L41.

[19] P. Howe, Pure Spinors Lines in Superspace and Ten-dimensional Supersymmetric Theories, Phys. Lett. B258 (1991) 141; Pure Spinors, Function Superspaces and Supergravity Theories in Ten-Dimensions and Eleven-Dimensions, Phys. Lett. B273 (1991) 90.

[20] N. Berkovits, Covariant Quantization of the Green-Schwarz Superstring in a Calabi-Yau Background, Nucl. Phys. B431 (1994) 258, arXiv:hep-th/9404162; A New Description of the Superstring, in Proceedings to VIII Jorge Swieca Summer School on particles and fields, p.490, World Scientific Publishing, 1996, [arXiv:hep-th/9604123]; Quantization of the Superstring with Manifest U(5) Super-Poincaré Covariance, Phys. Lett. B457 (1999) 94, arXiv:hep-th/9902099.

[21] E. R. Nissimov and S. J. Pacheva, Manifestly Superpoincaré Covariant Quantization of the Green-Schwarz Superstring, Phys. Lett. B202 (1988) 325.

E. Nissimov, S. Pacheva and S. Solomon, Covariant Canonical Quantization of the Green-Schwarz Superstring, Nucl. Phys. B297 (1988) 349.

[22] R. Kallosh and M. Rakhmanov, Covariant Quantization of the Green-Schwarz Superstring, Phys. Lett. B209 (1988) 233.

[23] I. A. Bandos and A. A. Zheltukhin, Spinor Cartan Moving N Hedron, Lorentz Harmonic Formulations of Superstrings, and Kappa Symmetry, JETP Lett. 54 (1991) 421 [Pisma Zh. Eksp. Teor. Fiz. 54 (1991) 421]; Green-Schwarz Superstrings in Spinor Moving Frame Formalism, Phys. Lett. B288 (1992) 77.

[24] N. Berkovits, Super-Poincaré Covariant Quantization of the Superstring, JHEP 0004 (2000) 018, arXiv:hep-th/0001035.

N. Berkovits and B. Carlini Valillo, Consistency of Super-Poincaré Covariant Superstring Tree Amplitudes, JHEP 0007 (2000) 015, [arXiv:hep-th/0004171].

N. Berkovits, Covariant Quantization of the Superstring, Int. J. Mod. Phys. A16 (2001) 
801, arXiv:hep-th/0008145.

N. Berkovits and O. Chandia, Lorentz Invariance of the Pure Spinor BRST Cohomology for the Superstring, Phys. Lett. B514 (2001) 394, arXiv:hep-th/0105149.

[25] N. Berkovits, Relating the RNS and Pure Spinor Formalism for the Superstring, JHEP 0108 (2001) 026, arXiv:hep-th/0104247.

[26] N. Berkovits, Cohomology in the Pure Spinor Formalism for the Superstring, JHEP 0009 (2000) 046, arXiv:hep-th/0006003.

N. Berkovits and O. Chandia, Massive Superstring Vertex Operator in D $=10$ Superspace, arXiv:hep-th/0204121.

G. Trivedi, Correlation Functions in Berkovits' Pure Spinor Formulation, arXiv:hepth/0205217].

[27] N. Berkovits, Covariant Quantization of the Superparticle Using Pure Spinors, JHEP 0109 (2001) 016, arXiv:hep-th/0105050]; Towards Covariant Quantization of the Supermembrane, arXiv:hep-th/0201151.

[28] N. Berkovits and V. Pershin, Supersymmetric Born-Infeld from the pure spinor formalism of the open superstring, hep-th/0205154.

[29] I. Oda and M. Tonin, On the Berkovits Covariant Quantization of GS Superstring, Phys. Lett. B520 (2001) 398, arXiv:hep-th/0109051.

[30] E. Witten, Topological Quantum Field Theory, Comm. Math. Phys. 117 (1988) 355; N. Berkovits and C. Vafa, On the Uniqueness of String Theory, Mod. Phys. Lett. A9 (1994) 653, arXiv:hep-th/9310170; $N=4$ Topological Strings, Nucl. Phys. B433 (1995) 123, arXiv:hep-th/9407190.

N. Berkovits, C. Vafa and E. Witten, Conformal Field Theories of AdS Background with Ramond-Ramond Flux, JHEP 9903 (1999) 018, arXiv:hep-th/9902098.

[31] I. Bandos and T. Bandos, Lorentz Harmonics and Superfield Action: $D=10, N=1$ Superstring, Class. Quant. Grav. 18 (2001) 1907 arXiv:hep-th/0010044.

[32] P. A. Grassi, G. Policastro, M. Porrati and P. van Nieuwenhuizen, Covariant Quantization of Superstrings without Pure Spinor Constraints, arXiv:hep-th/0112162.

P. A. Grassi, G. Policastro and P. van Nieuwenhuizen, The Massless Spectrum of Covariant Superstrings, arXiv:hep-th/0202123.

[33] W. Siegel, Classical Superstring Mechanics, Nucl. Phys. B263 (1986) 93.

[34] H. Ishikawa and M. Kato, Note on $N=0$ String as $N=1$ String, Mod. Phys. Lett. A9 (1994) 725, arXiv:hep-th/9311139.

F. Bastianelli, A Locally Supersymmetric Action for the Bosonic String, Phys. Lett. 
B322 (1994) 340, arXiv:hep-th/9311157.

N. Ohta and J. L. Petersen, N=1 from N=2 Superstrings, Phys. Lett. B325 (1994) 67, arXiv:hep-th/9312187.

M. Kato, Physical Spectra in String Theories: BRST Operators and Similarity Transformations, arXiv:hep-th/9512201.

[35] F. Bastianelli, N. Ohta and J. L. Petersen, A Hierarchy of Superstrings, Phys. Rev. Lett. 73 (1994) 1199, arXiv:hep-th/9403150.

[36] I. N. McArthur, Gauging of Nonlinearly Realized Symmetries, Nucl. Phys. B452 (1995) 456, arXiv:hep-th/9504160.

[37] N. Berkovits and P. Howe, Ten-Dimensional Supergravity Constraints from the Pure Spinor Formalism for the Superstring, arXiv:hep-th/0112160.

[38] A. Galperin and E. Sokatchev, A Twistor Formulation of the Nonheterotic Superstring with Manifest World Sheet Supersymmetry, Phys. Rev. D 48 (1993) 4810, arXiv:hepth/9304046].

[39] P. Pasti, D. P. Sorokin and M. Tonin, Superembeddings, Partial Supersymmetry Breaking and Superbranes, Nucl. Phys. B591 (2000) 109, arXiv:hep-th/0007048.

[40] P. S. Howe, O. Raetzel and E. Sezgin, On Brane Actions and Superembeddings, JHEP 9808 (1998) 011, arXiv:hep-th/9804051.

J. M. Drummond and P. S. Howe, Codimension Zero Superembeddings, Class. Quant. Grav. 18 (2001) 4477, [arXiv:hep-th/0103191].

P. S. Howe and U. Lindstrom, Kappa-Symmetric Higher Derivative Terms in Brane Actions, Class. Quant. Grav. 19 (2002) 2813, arXiv:hep-th/0111036. 